\title{
GMR
}

\section{Altered expression of metalloproteinase-2 and tissue inhibitor of metalloproteinase-2 in cervical disc herniation patients}

\author{
H.M. Zhuang', G.T. Xu' ${ }^{1}$, S.F. Wen ${ }^{2}$, Y.Y. Guo ${ }^{1}$ and Q. Huang ${ }^{1}$ \\ ${ }^{1}$ Department of Orthopedics, Jieyang People's Hospital, Jieyang, China \\ ${ }^{2}$ Department of Orthopedics, Guangzhou First People's Hospital, \\ Guangzhou, China \\ Corresponding author: H.M. Zhuang \\ E-mail: huanmingzhuang@sina.com
}

Genet. Mol. Res. 15 (2): gmr.15027594

Received September 9, 2015

Accepted December 4, 2015

Published April 26, 2016

DOI http://dx.doi.org/10.4238/gmr.15027594

\begin{abstract}
The aim of the current study was to examine matrix metalloproteinase-2 (MMP-2) and tissue inhibitor of metalloproteinase-2 (TIMP-2) expression in patients with cervical disc herniation (CDH). A total of 127 specimens from $\mathrm{CDH}$ patients undergoing posterior spinal surgery were obtained for the case group, which was divided into three subgroups: lateral protrusion $(\mathrm{N}=102)$, median protrusion $(\mathrm{N}=18)$, and paramedian protrusion $(\mathrm{N}=7)$. Another 55 specimens from subjects who had cervical spine trauma and underwent spinal canal decompression were obtained for the control group. Routine hematoxylin and eosin staining was performed for pathological diagnosis. Immunohistochemical (IHC) analysis was used to determine MMP-2 and TIMP-2 expression. Under light microscopy, MMP-2-positive cells presented brown-yellow or dark brown staining in the cell membrane or cytoplasm. MMP-2 expression in the case group was significantly higher than that in controls $(\mathrm{P}<0.05)$. Furthermore, MMP-2 expression in the lateral and median protrusion groups was significantly higher compared to that in the paramedian protrusion group (both $\mathrm{P}<0.05$ ),
\end{abstract}


while there was no apparent difference in MMP-2 expression between the lateral and median protrusion groups $(\mathrm{P}>0.05)$. IHC results showed that TIMP-2 expression in cases was significantly lower than that in controls $(\mathrm{P}<0.05)$. Spearman correlation analysis indicated that MMP2 was negatively correlated with TIMP-2 expression $(r=-0.418, \mathrm{P}<$ 0.001 ). In conclusion, MMP-2 expression increased, whereas TIMP2 expression decreased in CDH patients, suggesting that MMP-2 and TIMP-2 expression may contribute to $\mathrm{CDH}$ development.

Key words: Tissue inhibitor of metalloproteinase-2; Matrix metalloproteinase-2; Cervical disc herniation

\section{INTRODUCTION}

Cervical disc herniation $(\mathrm{CDH})$, which is most common in people in their thirties and forties, is a serious condition that occurs when the nucleus pulposus (NP), the component inside the intervertebral disc (IVD), extrudes into or protrudes through a compromised or incompetent annulus fibrosus (AF) (Hilibrand and Radcliff, 2011). In general, CDH can lead to neck and upper extremity pain, myelopathy, or radiculopathy resulting in numbness, weakness, or paresthesias (McCormick et al., 2003; Abbed and Coumans, 2007). Although the exact pathogenesis of $\mathrm{CDH}$ remains unclear, it is widely accepted that mechanical stress may be an important factor in the development of $\mathrm{CDH}$ and facet joint degeneration (Takahashi et al., 2013). It is well understood that the IVD performs key mechanical functions in distributing spinal load and maintaining flexibility (Loreto et al., 2011). The development of IVD degeneration may be largely attributed to important mediators of cell metabolism within the disc, which contribute to the synthesis, remodeling, and repair of the extracellular matrix (ECM) (Setton and Chen, 2006). Previous studies have documented that the metabolism of ECM components involves several participants such as matrix metalloproteinases (MMPs), aggrecanases, and tissue inhibitors of metalloproteinases (TIMPs) (Nagase and Kashiwagi, 2003; Visse and Nagase, 2003). As such, the expression of MMPs and TIMPs has recently been proposed to be closely related with the development of CDH (Zigouris et al., 2011; Vo et al., 2013).

MMPs are described as zinc-dependent endopeptidases that are capable of cleaving the protein components of the ECM, and thus have a relatively crucial role in tissue remodeling (Honkavuori-Toivola et al., 2013). As one important member of the MMP family, MMP-2 actively functions in the secondary breakdown of collagen during the tissue remodeling process (Kozaci et al., 2006; Séguin et al., 2008). Additionally, a large amount of evidence in animal models suggest that MMP-2 is significantly correlated with degenerative changes (Hsieh et al., 2009; Rastogi et al., 2013). It is widely accepted that IVD degeneration may be linked to proteolytic degradation of the ECM, resulting in several structural changes, such as AF disorganization, calcification of the cartilaginous end plates, and dehydration and fibrosis of the NP (Boos et al., 2002). MMP-2 was demonstrated to play an important part in degenerative disc disease as it interferes with the activity of AF cells (Rastogi et al., 2013). Particularly, the knockdown of MMP-2 in AF cells leads to a significantly decreased ability to reorganize and contract collagen gels (Rastogi et al., 2013). IVD tissue structure is thought to be mainly 
composed of collagens and proteoglycans (Kozaci et al., 2006). In the AF, matrix changes are accelerated with increase in age, which is thought to be a result of enhanced catabolic processing of the ECM (Akeda et al., 2007; Salo et al., 2008). While MMP-2 generally exists in an inactive pro-enzymatic form, it was observed that mechanical stress could induce MMP2 activation (MacLean et al., 2008; Rastogi et al., 2013). Since mechanical stress is a major risk factor for the development of $\mathrm{CDH}$, we hypothesized that MMP expression may be associated with the occurrence of $\mathrm{CDH}$. According to previous results, ECM remodeling by MMPs under the condition of pressure overload is largely mediated by TIMPs (Kassiri and Khokha, 2005; Spinale, 2007). The TIMP family proteins are the inhibitors of MMPs and consist of four members, namely TIMP-1, -2, -3, and -4 (Honkavuori-Toivola et al., 2013). Among them, TIMP-2 is specifically responsible for the regulation of MMP-2 activity (Têtu et al., 2006). Under normal conditions, TIMP-2 can activate pro-MMP-2 to MMP-2; however, when the amount of TIMP-2 is excessive, MMP-2 expression can then be repressed by TIMP2 (Brew and Nagase, 2010; Givvimani et al., 2013). Therefore, the goal of the present study was to measure the expression of MMP-2 and TIMP-2 in the cervical discs of CDH patients and normal controls and to further explore their potential roles in the pathogenesis of $\mathrm{CDH}$.

\section{MATERIAL AND METHODS}

\section{Ethics statement}

The current study protocol conformed to all relevant medical ethical guidelines, and was approved by the Ethics Committee of Jieyang People's Hospital. Written informed consent was obtained from all study participants and their families prior to their enrollment, and the study conformed to the principles of the Declaration of Helsinki (General Assembly of the World Medical Association, 2014).

\section{Study participants}

Between January 2012 and January 2013, a total of 182 cervical disc specimens were obtained from 182 patients in Jieyang People's Hospital. Among them, 127 specimens were obtained from $\mathrm{CDH}$ patients undergoing posterior spinal surgery, which comprised the case group. In this case group, there were 79 males and 48 females with an average age of $42.8 \pm$ 6.1 years (ranging from 27 to 59 years old). The selected patients were diagnosed with $\mathrm{CDH}$ by magnetic resonance imaging (MRI), and the diagnoses were confirmed by postoperative pathology as previously described (Mann et al., 2011). All CDH patients presented the following typical clinical and physical symptoms: 1) upper limb and arm pain, numbness, and/or lacking of strength; 2) weakness with walking or standing, lower limb numbness, and/or gait instability; 3 ) headache, nausea, dizziness, blurred vision and/or palpitation; and 4) neck and shoulder pain, and/or zonesthesia in the chest or abdomen. The radiographic manifestations of $\mathrm{CDH}$ were as follows: 1) conventional cervical anteroposterior and lateral radiographs as well as flexion/extension radiographs showed segmental instability, with the degree of degenerative narrowing of the intervertebral space being $<50 \%$ (compared to normal intervertebral space); and 2) hyperosteogeny in the anterior and posterior borders of the cervical vertebral body. All CDH patients further underwent MRI examination and were 
divided into the following three subgroups based on the results: 1) lateral protrusion group (N $=102)$; 2) median protrusion group $(\mathrm{N}=18)$; and 3) paramedian protrusion group $(\mathrm{N}=7)$. Additionally, another 55 specimens were obtained from subjects with cervical spine trauma who received spinal canal decompression, which comprised the control group. In this group, there were 37 males and 18 females, with an average age of $26.8 \pm 5.3$ years (ranging from 16 to 34 years). The preoperative X-ray and MRI findings of the controls revealed normal cervical intervertebral disc tissue as described at http://www.uscspine.com/conditions/neckherniated-disc.cfm. No differences in age, gender, or body mass index were observed between the $\mathrm{CDH}$ patients and controls. All the subjects included were negative for rheumatic diseases.

\section{Specimen collection and processing}

All samples were collected intraoperatively and were washed in normal saline solution. Then, the specimens were fixed in $10 \%$ formalin solution for $24 \mathrm{~h}$ at room temperature before dehydration and paraffin embedding. Subsequently, the paraffin embedded samples were cut into at least five 5- $\mu \mathrm{m}$ thick sections with a microtome (1516, Leica Microsystems, Nussloch, Germany) prior to routine hematoxylin and eosin (H\&E) staining for pathological diagnosis and immunohistochemical streptomycin-biotin-peroxidase (SP) staining for protein expression detection. A total of five sections from each tissue specimen were analyzed, of which one was selected for H\&E staining, two for SP staining of MMP-2 and TIMP-2, and two were used for negative controls.

\section{H\&E staining}

Routine H\&E staining was conducted as previously reported (Tschoeke et al., 2008). Briefly, the slides were dewaxed in xylene I ( 1 x $3 \mathrm{~min})$ and xylene II ( 1 x $3 \mathrm{~min})$ (AR, Guangdong Guanghua Chemical Factory Co., Ltd., China); hydrated in ethanol I (1 x 2 min) and ethanol II ( $1 \times 2 \mathrm{~min})$; and rehydrated in 95\% industrial alcohol (1 x $2 \mathrm{~min}), 85 \%$ alcohol ( $1 \times 2 \mathrm{~min}$ ), and $75 \%$ alcohol ( $1 \times 2 \mathrm{~min})$. After a short rinse with tap water for $3 \mathrm{~min}$, the deparaffinized tissue sections were stained with hematoxylin ( 1 x 8 min); washed with running tap water $(1 \times 3 \mathrm{~min})$ and $1 \%$ hydrochloric acid $(1 \times 30 \mathrm{~s})$; and rinsed with running tap water again $(1 \times 20 \mathrm{~min})$ and $1 \%$ ammonia $(1 \times 1 \mathrm{~min})$. Following a wash with running tap water for $30 \mathrm{~min}$, the slides were dyed with $0.5 \%$ eosin ( $1 \times 3 \mathrm{~min})$ and then dehydrated with $95 \%$ alcohol I and II for 2 min. Next, the sections were dehydrated with ethanol in graded alcohol solution similar to that described above. Finally, the slides were dipped in xylene (1 x $3 \mathrm{~min})$; washed in xylene I (1 x 2 min) and xylene II (1 x 2 min) until transparent; and then mounted with Permount (Fisher Scientific Co., Fair Lawn, NJ, USA) for viewing with an optical microscope (Olympus Optical, Tokyo, Japan).

\section{SP staining}

Immunohistochemical SP staining for detection of protein expression was performed in accordance with published procedures (Mazzoni et al., 2009). Briefly, the paraffin sections were dewaxed in $37^{\circ} \mathrm{C}$ xylene and hydrated with ethanol in graded alcohol solution, and then washed with phosphate-buffered saline (PBS, pH 7.4, 3 × 3 min). Thermal remediation for 
TIMP-2 was then performed using a citrate antigen retrieval solution. Specific procedures were as follows: first, $1500 \mathrm{~mL}$ citrate buffer solution, $\mathrm{pH}$ 6.0, was heated to boiling in a stainless steel pressure cooker. The tissue sections were then placed in the boiling buffer and heated at this constant temperature for $1 \mathrm{~min}$. After cooling to room temperature by rinsing with tap water, the sections were removed and washed with distilled water $(2 \times 3 \mathrm{~min})$ and PBS ( 2 x 3 min). After a drop of reagent A [peroxidase blocking solution (Dako)] was added, the pre-treated sections were incubated at room temperature for $10 \mathrm{~min}$. Following washes with PBS ( $3 \times 3 \mathrm{~min})$, reagent B (non-immune swine serum) was added and the sections were incubated at $37^{\circ} \mathrm{C}$ for $10 \mathrm{~min}$. After removal of the serum, $50 \mu \mathrm{L}$ primary mouse antihuman MMP-2 or TIMP-2 monoclonal antibodies purchased from Zhongshan Technology (Zhongshan, Beijing, China) was added and the sections were incubated at $4^{\circ} \mathrm{C}$ overnight. Following PBS washes ( 3 x 5 min), reagent C (biotin-labeled secondary antibody) was added, and the sections were then incubated at room temperature for $10 \mathrm{~min}$. After additional PBS washes ( $3 \times 3 \mathrm{~min}$ ), reagent $\mathrm{D}$ (streptavidin-peroxidase solution) was added, and the sections were then washed again with PBS ( 3 x 3 min). Next, 2 drops of 3,3'-diaminobenzidine tetra hydrochloride (Xiamen Engineering Machinery Company, Fujian, China) chromogen solution were added, and the sections were then rinsed with tap water for $3 \mathrm{~min}$. The slides were then counterstained with hematoxylin for $20 \mathrm{~s}$ and dried. Finally, the slides were mounted with Permount. Pathological sections provided by Zhongshan Technology known to be positive for MMP-2 and TIMP-2 were used as positive controls. Negative controls were treated with PBS instead of the primary antibody solution. Positive expression of MMP-2 and TIMP-2 was predefined as cells with brown-yellow or dark brown staining in the cell membrane or cytoplasm. A total of 10 non-overlapping fields of view were randomly selected and analyzed with a light microscope, and grayscale values were quantified.

\section{Statistical analysis}

All statistical analyses were performed using the SPSS version 19.0 statistical software (SPSS, Chicago, IL, USA). The nonparametric Wilcoxon test was used to evaluate if the data were normally distributed. Measurement data are reported as means \pm standard deviations

(means $\pm \mathrm{SD}$ ). Student $t$-tests were used to make comparisons between groups. All statistical tests were two-tailed, and $\mathrm{P}<0.05$ was considered statistically significant.

\section{RESULTS}

\section{MMP-2 protein expression}

Under light microscopy, cells positively expressing MMP-2 presented brown-yellow or dark brown staining in the cell membrane or cytoplasm. As shown in Table 1, the protein expression level of MMP-2 in the case group was higher than that in the control group, and there were significant differences in the grayscale values between the groups $(\mathrm{P}<0.05)$. Furthermore, the MMP-2 expression level in the lateral protrusion and median protrusion groups was significantly higher when compared to that in the paramedian protrusion group (both $\mathrm{P}<0.05$ ). Additionally, there was no apparent difference with respect to the expression of MMP-2 between the lateral protrusion group and median protrusion group ( $\mathrm{P}>0.05)$. Examples of MMP-2 staining in the case and control groups are presented in Figure 1. 
Table 1. Comparison of MMP-2 protein expression among the groups.

\begin{tabular}{l|c|c|c|c}
\hline & Cases (N) & Mean grayscale values of MMP-2 & $t$ & P \\
\hline Control group & 55 & $152.63 \pm 4.33$ & 22.13 & $<0.001^{\mathrm{a}}$ \\
\hline Case group & 127 & $169.52 \pm 4.89$ & 2.52 & $0.013^{\mathrm{b}, \mathrm{d}}$ \\
\hline Lateral protrusion group & 102 & $168.84 \pm 5.01$ & 3.20 & $0.004^{\mathrm{c}}$ \\
\hline Median protrusion group & 18 & $170.55 \pm 4.62$ & & \\
\hline Paramedian protrusion group & 7 & $163.93 \pm 4.71$ & & \\
\hline
\end{tabular}

MMP-2 = matrix metalloproteinase-2. a Comparison of MMP-2 expression level between the case group and the control group. ${ }^{\mathrm{b} C o m p a r i s o n}$ of MMP-2 expression level between the lateral protrusion group and the paramedian protrusion group. ${ }^{\circ}$ Comparison of MMP-2 expression level between the median protrusion group and the paramedian protrusion group. ${ }^{\mathrm{d}}$ Comparison of MMP-2 expression level between the lateral protrusion group and the median protrusion group.

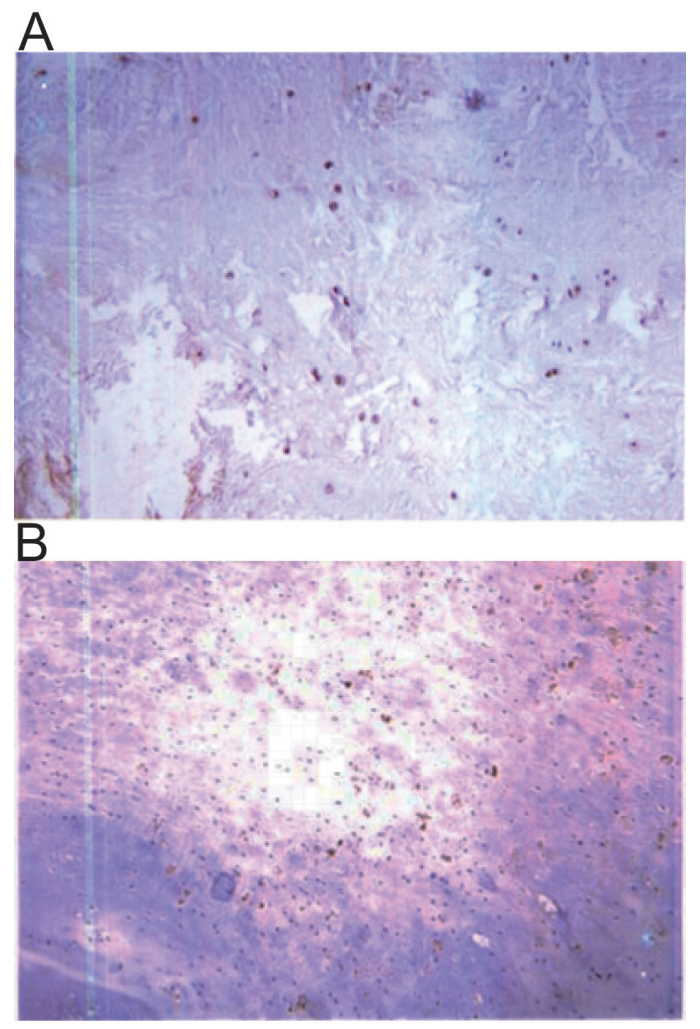

Figure 1. MMP-2 detection using immunohistochemical streptomycin-biotin-peroxidase staining as seen under light microscopy (100X) in the case group and the control group. A. Positive expression of MMP-2 in the case group. B. Positive expression of MMP-2 in the control group.

\section{TIMP-2 protein expression comparison}

Positive expression of TIMP-2 was observed as brown-yellow or dark brown staining in the cell membrane or cytoplasm under light microscopy. Examples of TIMP-2 staining in the case and control groups are presented in Figure 2. The SP-staining results revealed that the protein expression level of TIMP-2 in the case group was significantly lower than that in the control group $(161.37 \pm 3.99$ and $168.53 \pm 4.59$, respectively; $t=10.61, \mathrm{P}<0.001)$. 


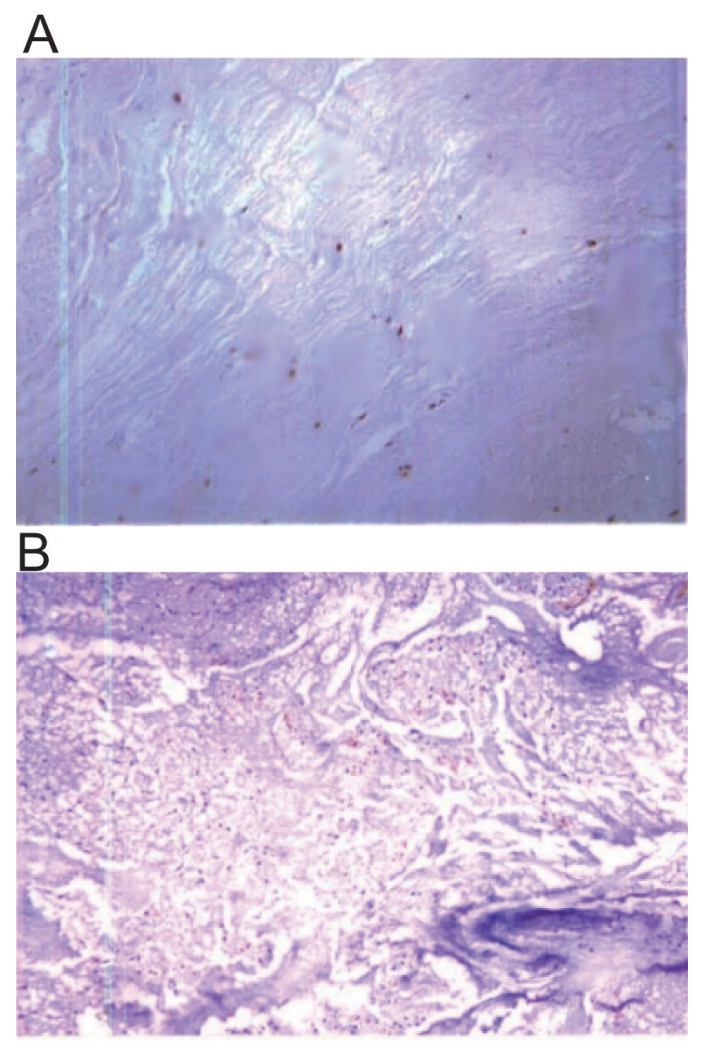

Figure 2. TIMP-2 detection using immunohistochemical streptomycin-biotin-peroxidase staining as seen under light microscopy (100X) in the case group and the control group. A. Positive expression of TIMP-2 in the case group. B. Positive expression of TIMP-2 in the control group.

\section{Correlation analysis between MMP-2 and TIMP-2}

Next, a correlation analysis between MMP-2 and TIMP-2 expression in the cervical disc specimens was carried out. The Spearman correlation analysis results indicated that there was a significantly negative correlation between the expression level of MMP-2 and that of TIMP-2 in patients with $\mathrm{CDH}(r=-0.418, \mathrm{P}<0.001)$. These results demonstrate that reduced expression of TIMP-2 may be a potential factor contributing to the upregulation of MMP-2 expression.

\section{DISCUSSION}

In the current study, the expression of MMP-2 and TIMP-2 in the cervical discs from both $\mathrm{CDH}$ patients and non-CDH controls was carefully and systematically assessed. We found that the $\mathrm{CDH}$ case group had significantly higher expression of MMP-2 than the control group; moreover, the expression of MMP-2 in CDH patients with lateral and median protrusions was higher than that of patients with paramedian protrusions. These results demonstrate that 
increased expression of MMP-2 may be involved in the development of CDH. It has previously been reported that ECM degradation is a feature of IVD degeneration (Yurube et al., 2012). Additionally, MMPs are capable of breaking down certain ECM proteins and thus have an active impact on ECM remodeling via the degradation of matrix scaffold macromolecules (Nissi et al., 2013). MMP-2 belongs to the MMP family, and plays an important role in ECM degradation both directly and indirectly (Kozaci et al., 2006). It is well known that water and collagen are the major components of the IVD, while the ECM of the NP and AF is mainly composed of type 2 collagens (Yurube et al., 2012; Vo et al., 2013). There is also evidence showing that several changes in the NP may lead to abnormal load in the AF, causing further breakdown and finally resulting in structural failure found in IVD degeneration (Vo et al., 2013). Along these lines, MMP-2 has been observed to participate in the secondary processing of collagen and was found to effectively cleave intact triple-helical collagen (Rastogi et al., 2013). More importantly, it was demonstrated that MMP-2 knockdown reduced localized gelatin degradation and repressed the ability of AF cells to remodel the collagen matrix (Rastogi et al., 2013). Considering these results, it is plausible that increased MMP-2 expression may be linked to the pathogenesis of $\mathrm{CDH}$.

Another important result of the current study was that the expression of TIMP-2 in the case group was significantly lower than that of the control group, indicating that reduced expression of TIMP-2 may also be associated with CDH pathogenesis. It has been suggested that the process of tissue remodeling, which is characterized by rapid growth and structural tissue changes, involves both the function of MMPs and their inhibitors, namely TIMPs (Nissi et al., 2013). TIMP-2 is considered a unique member of the TIMP family since it not only suppresses MMP-2, but also promotes the cell-surface activation of pro-MMP-2 via the interaction with MT1-MMP (Stetler-Stevenson, 2008). Thus, TIMP-2 exhibits dual functions as it has the ability to activate pro-MMP-2 and inhibit active MMP-2 at the same time (StetlerStevenson, 2008; Givvimani et al., 2013). Specifically, under normal conditions, TIMP-2 is responsible for the activation of pro-MMP-2, but when the amount of TIMP-2 is too high, MMP-2 is consequently repressed by TIMP-2 (Brew and Nagase, 2010). As discussed above, the occurrence of $\mathrm{CDH}$ may be partly attributed to increased expression of MMP-2 owing to its involvement in ECM degradation. Therefore, we hypothesize that reduced expression of TIMP-2 may lead to the upregulation of MMP-2 expression, thereby contributing to the etiology of CDH.

In summary, the results of the current study showed that MMP-2 expression significantly increased, whereas TIMP-2 expression significantly decreased in patients with $\mathrm{CDH}$, suggesting that MMP-2 and TIMP-2 expression may contribute to the development of CDH. Thus, the measurement of MMP-2 and TIMP-2 expression may be important in the diagnosis of $\mathrm{CDH}$.

\section{Conflicts of interest}

The authors declare no conflict of interest.

\section{ACKNOWLEDGMENTS}

We would like to acknowledge the Department of Orthopedics, Jieyang People's Hospital and Guangzhou First People's Hospital for their helpful comments on this paper. 


\section{REFERENCES}

Abbed KM and Coumans JV (2007). Cervical radiculopathy: pathophysiology, presentation, and clinical evaluation. Neurosurgery 60 (Supp1 1): S28-S34. http://dx.doi.org/10.1227/01.NEU.0000249223.51871.C2

Akeda K, An HS, Pichika R, Patel K, et al. (2007). The expression of NG2 proteoglycan in the human intervertebral disc. Spine 32: 306-314. http://dx.doi.org/10.1097/01.brs.0000254108.08507.04

Boos N, Weissbach S, Rohrbach H, Weiler C, et al. (2002). Classification of age-related changes in lumbar intervertebral discs: 2002 Volvo Award in basic science. Spine 27: 2631-2644. http://dx.doi.org/10.1097/00007632-200212010$\underline{00002}$

Brew K and Nagase H (2010). The tissue inhibitors of metalloproteinases (TIMPs): an ancient family with structural and functional diversity. Biochim. Biophys. Acta 1803: 55-71. http://dx.doi.org/10.1016/j.bbamcr.2010.01.003

General Assembly of the World Medical Association (2014). World Medical Association Declaration of Helsinki: ethical principles for medical research involving human subjects. J. Am. Coll. Dent. 81: 14-18.

Givvimani S, Kundu S, Narayanan N, Armaghan F, et al. (2013). TIMP-2 mutant decreases MMP-2 activity and augments pressure overload induced LV dysfunction and heart failure. Arch. Physiol. Biochem. 119: 65-74. http://dx.doi.org/ $\underline{10.3109 / 13813455.2012 .755548}$

$<$ edb>Hilibrand AS and Radcliff K (2011). Cervical Disk Herniation. In: Musculoskeletal examination of the spine: Making the complex simple (Rihn JA and Harris EB, eds). SLACK, Thorofare, NJ, 63.</edb>

Honkavuori-Toivola M, Santala M, Soini Y, Turpeenniemi-Hujanen T, et al. (2013). Combination of strong MMP-2 and weak TIMP-2 immunostainings is a significant prognostic factor in endometrial carcinoma. Dis. Markers 35: 261266. http://dx.doi.org/10.1155/2013/416870

Hsieh AH, Hwang D, Ryan DA, Freeman AK, et al. (2009). Degenerative anular changes induced by puncture are associated with insufficiency of disc biomechanical function. Spine 34: 998-1005. http://dx.doi.org/10.1097/ BRS.0b013e31819c09c4

Kassiri Z and Khokha R (2005). Myocardial extra-cellular matrix and its regulation by metalloproteinases and their inhibitors. Thromb. Haemost. 93: 212-219.

Kozaci LD, Guner A, Oktay G and Guner G (2006). Alterations in biochemical components of extracellular matrix in intervertebral disc herniation: role of MMP-2 and TIMP-2 in type II collagen loss. Cell Biochem. Funct. 24: 431-436. http://dx.doi.org/10.1002/cbf.1250

Loreto C, Musumeci G, Castorina A, Loreto C, et al. (2011). Degenerative disc disease of herniated intervertebral discs is associated with extracellular matrix remodeling, vimentin-positive cells and cell death. Ann. Anat. 193: 156-162. http://dx.doi.org/10.1016/j.aanat.2010.12.001

MacLean JJ, Roughley PJ, Monsey RD, Alini M, et al. (2008). In vivo intervertebral disc remodeling: kinetics of mRNA expression in response to a single loading event. J. Orthop. Res. 26: 579-588. http://dx.doi.org/10.1002/jor.20560

Mann E, Peterson CK and Hodler J (2011). Degenerative marrow (modic) changes on cervical spine magnetic resonance imaging scans: prevalence, inter- and intra-examiner reliability and link to disc herniation. Spine 36: 1081-1085. http://dx.doi.org/10.1097/BRS.0b013e3181ef6a1e

Mazzoni A, Pashley DH, Tay FR, Gobbi P, et al. (2009). Immunohistochemical identification of MMP-2 and MMP-9 in human dentin: correlative FEI-SEM/TEM analysis. J. Biomed. Mater. Res. A 88: 697-703. http://dx.doi.org/10.1002/ jbm.a.31920

McCormick WE, Steinmetz MP and Benzel EC (2003). Cervical spondylotic myelopathy: make the difficult diagnosis, then refer for surgery. Cleve. Clin. J. Med. 70: 899-904. http://dx.doi.org/10.3949/ccjm.70.10.899

Nagase H and Kashiwagi M (2003). Aggrecanases and cartilage matrix degradation. Arthritis Res. Ther.5: 94-103.PubMed http://dx.doi.org/10.1186/ar630

Nissi R, Talvensaari-Mattila A, Kotila V, Niinimäki M, et al. (2013). Circulating matrix metalloproteinase MMP-9 and MMP-2/TIMP-2 complex are associated with spontaneous early pregnancy failure. Reprod. Biol. Endocrinol. 11: 2. http://dx.doi.org/10.1186/1477-7827-11-2

Rastogi A, Kim H, Twomey JD and Hsieh AH (2013). MMP-2 mediates local degradation and remodeling of collagen by annulus fibrosus cells of the intervertebral disc. Arthritis Res. Ther. 15: R57. http://dx.doi.org/10.1186/ar4224

Salo J, Mackiewicz Z, Indahl A, Konttinen YT, et al. (2008). Plasmin-matrix metalloproteinase cascades in spinal response to an experimental disc lesion in pig. Spine 33: 839-844. http://dx.doi.org/10.1097/BRS.0b013e31816b1f1d

Séguin CA, Pilliar RM, Madri JA and Kandel RA (2008). TNF-alpha induces MMP2 gelatinase activity and MT1-MMP expression in an in vitro model of nucleus pulposus tissue degeneration. Spine 33: 356-365. http://dx.doi.org/10.1097/ BRS.0b013e3181642a5e

Setton LA and Chen J (2006). Mechanobiology of the intervertebral disc and relevance to disc degeneration. J. Bone Joint

Genetics and Molecular Research 15 (2): gmr.15027594

CFUNPEC-RP www.funpecrp.com.br 
Surg. Am. 88 (Suppl 2): 52-57. http://dx.doi.org/10.2106/JBJS.F.00001

Spinale FG (2007). Myocardial matrix remodeling and the matrix metalloproteinases: influence on cardiac form and function. Physiol. Rev. 87: 1285-1342. http://dx.doi.org/10.1152/physrev.00012.2007

Stetler-Stevenson WG (2008). The tumor microenvironment: regulation by MMP-independent effects of tissue inhibitor of metalloproteinases-2. Cancer Metastasis Rev. 27: 57-66. http://dx.doi.org/10.1007/s10555-007-9105-8

Takahashi Y, Yasuhara T, Kumamoto S, Yoneda K, et al. (2013). Laterality of cervical disc herniation. Eur. Spine J. 22: 178-182. http://dx.doi.org/10.1007/s00586-012-2565-8

Têtu B, Brisson J, Wang CS, Lapointe H, et al. (2006). The influence of MMP-14, TIMP-2 and MMP-2 expression on breast cancer prognosis. Breast Cancer Res. 8: R28. http://dx.doi.org/10.1186/bcr1503

Tschoeke SK, Hellmuth M, Hostmann A, Robinson Y, et al. (2008). Apoptosis of human intervertebral discs after trauma compares to degenerated discs involving both receptor-mediated and mitochondrial-dependent pathways. J. Orthop. Res. 26: 999-1006. http://dx.doi.org/10.1002/jor.20601

Visse R and Nagase H (2003). Matrix metalloproteinases and tissue inhibitors of metalloproteinases: structure, function, and biochemistry. Circ. Res. 92: 827-839. http://dx.doi.org/10.1161/01.RES.0000070112.80711.3D

Vo NV, Hartman RA, Yurube T, Jacobs LJ, et al. (2013). Expression and regulation of metalloproteinases and their inhibitors in intervertebral disc aging and degeneration. Spine J. 13: 331-341.http://dx.doi.org/10.1016/j.spinee.2012.02.027

Yurube T, Takada T, Suzuki T, Kakutani K, et al. (2012). Rat tail static compression model mimics extracellular matrix metabolic imbalances of matrix metalloproteinases, aggrecanases, and tissue inhibitors of metalloproteinases in intervertebral disc degeneration. Arthritis Res. Ther. 14: R51.PubMed http://dx.doi.org/10.1186/ar3764

Zigouris A, Batistatou A, Alexiou GA, Pachatouridis D, et al. (2011). Correlation of matrix metalloproteinases-1 and -3 with patient age and grade of lumbar disc herniation. J. Neurosurg. Spine 14: 268-272. http://dx.doi. org/10.3171/2010.9.SPINE09935 